\title{
Rupture of Interstitial Pregnancy in Early Gestation due to Prostaglandin Abuse
}

\author{
SANDEEP KOUR ${ }^{1}, \mathrm{KAMLESH} \mathrm{MANHAS}^{2}$
}

\begin{abstract}
:
Interstitial pregnancy occurs when the fertilised ovum implants in the interstitial portion of the fallopian tube. It occurs in around $2 \%$ of all the ectopic pregnancies. It can last up to mid trimester before it ruptures spontaneously as it can grow inside the interstitium for long period of time. If not detected early, can lead to a fatal outcome. Mifepristone (antiprogesterone) followed by a low dose of misoprostol (prostaglandin E1 analog) has been used for termination of early pregnancy after ruling out ectopic pregnancy, but there have been many reports of misuse of this combination especially in the developing countries. Here we report a case of interstitial pregnancy, which ruptured at 6 weeks of gestation due to misuse of this combination as an over the counter drug without looking for ectopic pregnancy.
\end{abstract}

Key-words: Ectopic pregnancy, interstitial pregnancy, misoprostol misuse, mifepristone misuse.

\section{Introduction:}

Interstitial pregnancy is a rare occurrence and difficult to diagnose ${ }^{1}$. Spontaneous rupture of interstitial pregnancy usually occurs in the second trimester because of greater distensibility of the myometrium covering the tube ${ }^{2}$. A number of cases of rupture of interstitial pregnancy have been reported in the literature ${ }^{3-5}$. We report a case of ruptured interstitial pregnancy in the first trimester due to misuse of oral prostaglandins and mifepristone. Our case highlights the easy availability of oral prostaglandins and mifepristone combination as over the counter (OTC) drugs dispensed by drug retailers without the doctor's prescription and dangers of using this combination without looking for ectopic pregnancy.

\section{Case Report:}

A 32-year-old para 1, not booked for antenatal care presented to the out patient clinic with complaints of left lower abdominal pain of 7 days. Her previous delivery two years ago had been conducted by cesarean section for pregnancy-induced hypertension.

Five days after missing her periods she had got her pregnancy test done which turned out to be positive. A drug retailer dispensed her tablets of mifepristone and misoprostol without any prescription and she took two tablets of mifepristone (200 mg each) followed by a single tablet of misoprostol (200 microgram each) after 48 hours. Four days after taking the misoprostol she had vaginal bleeding without expulsion of any products. Bleeding lasted for 5-6 days. Two weeks after having taken the drugs she had an acute episode of pain left iliac region, which lasted few hours, without any syncope. An ultrasound examination done at the advice of local physician did not reveal a gestational sac in the uterine cavity and normal adnexae. However

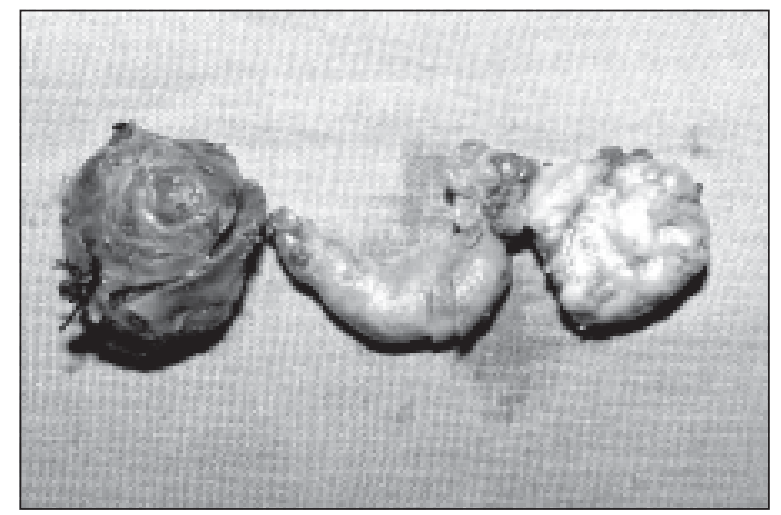

Figure: Photograph of the resected specimen showing left cornue of the uterus along with fallopian tube and ovary

1. Assistant Professor, Department of Obstetrics and Gynecology, Acharaya Sri Chander College of Medical Sciences, Sidhra, Jammu, Jammu \& Kashmir India

2. Professor, Department of Obstetrics and Gynecology, Acharaya Sri Chander College of Medical Sciences, Sidhra, Jammu , Jammu \& Kashmir India 
she had free fluid in the pouch of Douglas and she was referred to our department.

Physical examination revealed pallor and she had tachycardia with a pulse rate of 112 beats per minute and blood pressure of $130 / 90 \mathrm{mmHg}$. Bimanual examination revealed enlarged uterus corresponding to 6-8 weeks of gestation with irregular contour and tenderness in both the fornices. Her hemoglobin was $7 \mathrm{~g} / \mathrm{dl}$. A diagnosis of ruptured ectopic pregnancy was made and patient was taken for emergency exploratory laparotomy. At laparotomy she had about 1.5 liters of blood in the abdominal cavity, uterus was enlarged with a bulge on the left side of fundus revealing ruptured ectopic gestational sac. Left fallopian tube was edematous and friable with multiple cysts in the ovary. Wedge resection of the left cornue along with left salphingo-oophrectomy was done. It was found to be an interstitial pregnancy occupying region between round ligament and fallopian tube evidenced by the presence of gestational sac which was confirmed by histopathological examination. The ovary was removed as it was found to have multiple cysts.

\section{Discussion:}

Interstitial pregnancy is a rare occurrence, occurring in around $2 \%$ of all the ectopic pregnancies ${ }^{1}$. It can last up to mid trimester before it ruptures spontaneously or during induction of abortion ${ }^{[2]}$ and early detection is required to prevent a fatal outcome. In our patient the rupture occurred at 6 weeks of gestation, the reason of which appears to be misuse of abortificient in view of the temporal relation between the drug intake and rupture of interstitial pregnancy. Spontaneous rupture is also more likely to occur in the second trimester.

Mifepristone has anti progesterone activity, which is because of competitive interaction with progesterone at the progesterone receptor site $^{6}$. Mifepristone is indicated for medical termination of pregnancy up to 49 days of pregnancy and is contraindicated with a confirmed or suspected ectopic pregnancy. Ultrasound scan needs to be done prior to administration of the drug if ectopic pregnancy cannot be ruled out clinically. Misoprostol is a prostaglandin E1 analog that was initially used for the prevention of NSAID-induced gastric ulcers. Due to its uterotonic properties it was abused to induce illegal abortion in the late 1980s. Presently misoprostol has become one of the most effective drugs for terminating pregnancies in the first and second trimesters but there are reports of its misuse from the underdeveloped world where these drugs can be bought without medical prescription $7,8,9,10$.

Our case is a rare occurrence of interstitial pregnancy rupturing at 6 weeks of gestation due to abortificients abuse, which are sometimes dispensed by the drug retailers without a prescription by the physician. The present case emphasises the need to rule out ectopic pregnancy before using mifepristone and misoprostol for termination of pregnancy. There is need for proper monitoring and to implement the regulations for dispensing of abortificients. This can prevent their misuse and possible morbidity and mortality.

\section{Conclusion:}

Interstitial pregnancy is a rare occurrence and is seen in only two percent of all the ectopic pregnancies and can have a fatal outcome if missed. There is a widespread misuse of oral abortificients in our country and these drugs are sometimes dispensed by the drug retailers without a doctor's prescription. There should be better implementation of regulation of dispensing drugs. In patients who are prescribed oral abortificients it is important that interstitial pregnancy be ruled out by ultrasound examination and if necessary by transvaginal ultrasound, if possible. All clients should be counselled before prescribing miferpristone misoprostol that they should report to hospital if there is unusual/severe pain in lower abdomen

\section{References:}

1. J.Bouyer, J.Coste1, H.Fernandez, J.L.Pouly, and N.Job-Spira1. Sites of ectopic pregnancy a 10 year population-based study of 1800 cases. Human Reproduction.2002; 17: 3224- 3230.

2. Petersen KR, Larsen GK, Norring K, Jensen FR. Misdiagnosis of interstitial pregnancy followed by uterine cornual rupture during induced midtrimester abortion. Acta Obstet Gynecol Scand. 1992;71:316-8.

3. Tulandi T, Al-Jaroudi D. Interstitial pregnancy: results generated from the Society of Reproductive Surgeons Registry. Obstet Gynecol. $2004 ; 103$ : 47-50 
4. Chan LY, Fok WY, Yuen PM. Pitfalls in diagnosis of interstitial pregnancy. Acta Obstet Gynecol Scand. 2003 ;82:867-70

5. Habek D, Mrcela M, Rubin M, Hrgovic Z.Ruptured interstitial pregnancy Two case reports. Arch Gynecol Obstet. 2003; 267:170-2.

6. Baulieu EE. RU 486 (mifepristone). A short overview of its mechanisms of action and clinical uses at the end of 1996. Ann N Y Acad Sci. 1997; 828:47-58.

7. Chong YS, Su LL, Arulkumaran S. Misoprostol: a quarter century of use, abuse, and creative misuse. Obstet Gynecol Surv. 2004; 59:128-40.
8. Schuler L, Pastuszak A, Sanseverino TV et al. Pregnancy outcome after exposure to misoprostol in Brazil: a prospective, controlled study. Reprod Toxicol. 1999;13:147-51

9. Gonzalez CH, Marques-Dias MJ, Kim CA et al. Congenital abnormalities in Brazilian children associated with misoprostol misuse in first trimester of pregnancy. Lancet. 1998; 30; 351:1624-7.

10. Coelho HL, Teixeira AC, Santos AP et al. Misoprostol and illegal abortion in Fortaleza, Brazil. Lancet. 1993; 341:1261-3. 